\title{
Self-Organizing Construction Method of Offshore Structures by Cellular Automata Model
}

\author{
Takeshi Ishida \\ Department of Ocean Mechanical Engineering, National Fisheries University, 2-7-1 Nagatahonmachi, Shimonoseki 759-6595, Japan \\ Correspondence should be addressed to Takeshi Ishida; ishida@fish-u.ac.jp
}

Received 21 November 2014; Accepted 23 February 2015

Academic Editor: Azah Mohamed

Copyright (C) 2015 Takeshi Ishida. This is an open access article distributed under the Creative Commons Attribution License, which permits unrestricted use, distribution, and reproduction in any medium, provided the original work is properly cited.

\begin{abstract}
We propose a new algorithm to build self-organizing and self-repairing marine structures on the ocean floor, where humans and remotely operated robots cannot operate. The proposed algorithm is based on the one-dimensional cellular automata model and uses simple transition rules to produce various complex patterns. This cellular automata model can produce various complex patterns like sea shells with simple transition rules. The model can simulate the marine structure construction process with distributed cooperation control instead of central control. Like living organism is constructed with module called cell, we assume that the self-organized structure consists of unified modules (structural units). The units pile up at the bottom of the sea and a structure with the appropriate shape eventually emerges. Using the attribute of emerging patterns in the one-dimensional cellular automata model, we construct specific structures based on the local interaction of transition rules without using complex algorithms. Furthermore, the model requires smaller communication data among the units because it only relies on communication between adjacent structural units. With the proposed algorithm, in the future, it will be possible to use self-assembling structural modules without complex built-in computers.
\end{abstract}

\section{Introduction}

Most existing marine structures are of floating type, whereas very few are fixed to the seabed, for example, gravity-based platforms, compliant structures, and jacket structures. Such fixed structures are constructed in relatively shallow seas. The deepest jacket structure is at a depth of $411 \mathrm{~m}$ in the Gulf of Mexico and the deepest compliant structure is at a depth of $1,219 \mathrm{~m}$. With the present engineering capabilities, the construction of deep-seabed marine structures deeper than $2,000 \mathrm{~m}$ is considered extremely difficult.

Recently, the need to exploit ocean floor resources, such as rare-earth deposits or methane hydrates, is strong and increasing. In fact, there are some articles which reported the existence of rich distribution of rare metal resources around the area of hydrothermal deposit of oceans bed [1]. However, these deep hydrothermal deposits are distributed more than 1000-3000 m deep, which hinders the efforts to reach them from sea surface platforms. Thus, inevitably, structures have to be constructed at the deep sea floor for large-scale exploitation of such resources. Furthermore, it is useful to create control points of fisheries at the seabed level to better manage the spatial distribution of such resources. This technology may be applied to the construction of marine farm in the future.

However, there are many challenges to construct these marine bases, as below.

(1) It is impossible for humans to build an offshore structure directly in the deep sea beyond a certain depth, and it is limited to use manned submersible. Robot ROV (remotely operated vehicle) controlled from surface ship is also the alternative measure, but the activity range of ROV on ocean floor is limited.

(2) Electric wave does not reach deep under sea, and also supersonic wave limited the capacity and distance of communication. Remote control with the radio from the sea surface is impossible. 
(3) Energy supply is another problem. The capacity of battery use for equipment or robot working on ocean bed is limited. Therefore it is necessary to provide electricity from sea surface or offshore land; in this case energy loss is inevitable during electric power transmission to deep ocean bed. Therefore, it is necessary to build the structure which does not use energy as much as possible.

(4) It is difficult to conduct maintenance of units which once are built on ocean bed from the viewpoint of physical work, communication, and energy.

One of the methods to solve these difficulties is autonomous robot that can work independently, judge own movement, and return to the surface for charging. If many autonomous robots are used under decentralized cooperation control, it is possible to build self-organizing marine structure. Furthermore, learning from natural phenomena, such as biological morphosis or self-repairing function, is one of the efficient methods for construction of self-organizing structure. As for the living organism, basic units called cells build an organ and body based on intracellular genetic information, and also they have the ability to self-repair. From the analogy of living organism, it is ideal that a self-assembling marine structure is automatically constructed with structural units, which are similar to a biological cell, under decentralized cooperation control.

Considering the abovementioned difficulties, we summarize the required conditions for self-organizing marine structures. (1) To avoid the complexity of using autonomous robots, it is necessary to build the structure using same-shape structural units. (2) All structural units have the blueprint of the entire marine structure, like genetic information within cell, for decentralized cooperation and control. (3) It is difficult to provide electricity to deep marine structure from the sea surface; moreover, it is hard to periodically change the batteries. Therefor it is impossible to load computer which consumes a lot of electricity like recent PC, and structural units need information-processing circuit that consumes as little electricity as possible.

Presently, research on self-organizing marine structures is lacking. Nonetheless, several papers have mentioned selforganization methods to construct space stations and moon bases [2]. Furthermore, there is considerable work on selforganizing robots that are used to produce specific shapes by connecting module units automatically. One of the early examples is "Fractam" by Murata $[3,4]$. There is no autonomous robot for construction of marine structure as it is now.

Past reports on self-organizing robots concluded that it was difficult to find the appropriate algorithm for constructing a specific shape by unifying interacting modules. In other words, an impasse is almost certain. Furthermore, these difficulties depend on identifying each module or depend on the existence of a leader module. It is relatively easy to create specific shapes with modules that have an ID number and a module leader. On the other hand, the probability of successful completion of a shape decreases when using modules without an ID number and a module leader. To create more sophisticated algorithms, the self-organization phenomena,

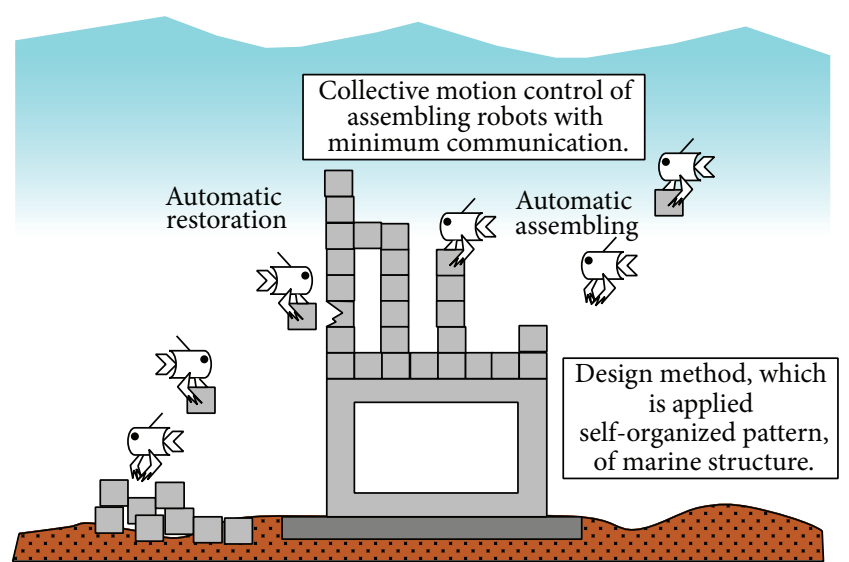

Figure 1: Outline of a self-organizing marine structure. The unified modules (structural units) are piled on a basement at the bottom of the sea and eventually the structure with the appropriate shape emerges. It is assumed that each module only communicates with adjacent units. Using the attribute of emerging pattern in onedimensional cellular automata, specific structures based on the local interaction of transition rules without using complex algorithms can be constructed.

such as Turing patterns or the Belousov-Zhabotinsky reaction, are likely needed.

We propose a new algorithm for building submarine structures under deep-sea conditions where humans or ROVs cannot operate. The algorithm is based on decentralized cooperation control without a control center for building selforganizing and self-repairing marine structures. Like living organism is constructed with module called cell, we assumed that our self-organized structure consists of unified module (structural unit). The units are piled on a basement at the bottom of the sea and eventually the structure with the appropriate shape emerges. It is assumed that each unit only communicates with adjacent units.

Generally, it is difficult to collect underwater units for repairs. Furthermore, it is difficult to provide the units with electricity; thus, it is desirable to use simple circuits without electricity. We assume that each unit contains the simplest circuit without a CPU which consumes large amounts of electricity. Figure 1 shows the outline of the self-organizing marine structure.

As mentioned above, it is necessary to use self-organization phenomena for successfully constructing specific shapes. To model the structure in Figure 1, we use a one-dimensional cellular automaton. A cellular automaton is a discretized model of space and time and simulates various physical phenomena and the state of the cellular grid transits is based on the transition rules which are described in a form to refer to adjacent grids. One-dimensional cellular automata studied by Wolfram showed some type of shell pattern emerging with specific transition rules [5].

Using the attribute of emerging pattern in one-dimensional cellular automata, specific structures based on the local interaction of transition rules without using complex algorithms can be constructed. With the proposed method, it will 
be possible to build self-assembling structural units without built-in complex computers. The proposed research fills the current gap in the literature of self-organization algorithms for building marine structures.

\section{Model}

2.1. Underwater Self-Organized Structure. The marine structure model has the following characteristics. (1) The structure is built on a foundation on the ocean bed. (2) The structure consists of unified modules (structural units) that are piled up and connected to each other. (3) Each unit contains the design information which determines the final shape of the marine structure, and each unit evaluates the conditions using information from the adjacent units and the internal design information. (4) We assume that autonomous robots carry the necessary units to the top of the structure and focus on the construction algorithm; the details of the carrier robots are not specified because the algorithm for the carrier robot(s) is an additional problem. (5) We assume that there is no central control point and that the structure is constructed using a decentralized cooperation algorithm. (6) Each unit does not have an ID number and can be any part of the structure. (7) Each unit only communicates with adjacent units; thus, it shares information neither with remote units nor with the units of the entire structure.

In the conventional algorithms, to achieve autonomous complete construction with layer stacking, it is necessary for each unit to contain the entire blueprint. Computer programs for this type of algorithms must compare the coordinates of each unit to the blueprint information and determine connections. The problem is that each unit must contain huge amounts of data concerning each unit potion. This is further exacerbated in large marine structures, which require the use of computers with large memory capacity in each module. Considering the difficulties in providing electricity to the equipment at the ocean bed, the use of such computer power in each module is impractical.

In contrast, the proposed method can decrease the amount of blueprint data and intermodule communication using one-dimensional cellular automata. Living creatures use self-organization to construct their bodies. For example, the positions of all cells are not recorded in genes, and all chemical reactions inside of cell are not controlled in brain. It is thought that living creatures can reduce the amount of genetic information by taking advantage of self-organization.

If it is an extremely simple shape (e.g., modules piling up linearly), then the design information can be simplified. On the other hand, it is difficult to apply the rules for simple structures to the more complex marine structures.

\subsection{One-Dimensional Cellular Automata Model. The one-} dimensional cellular automata model is a discrete model where the next cell state is determined by the states of the adjacent cells and the state of the cell. Figure 2 shows the outline of the one-dimensional cellular automata model. In this figure, the horizontal direction denotes space and the vertical direction denotes time. Furthermore, periodic boundary

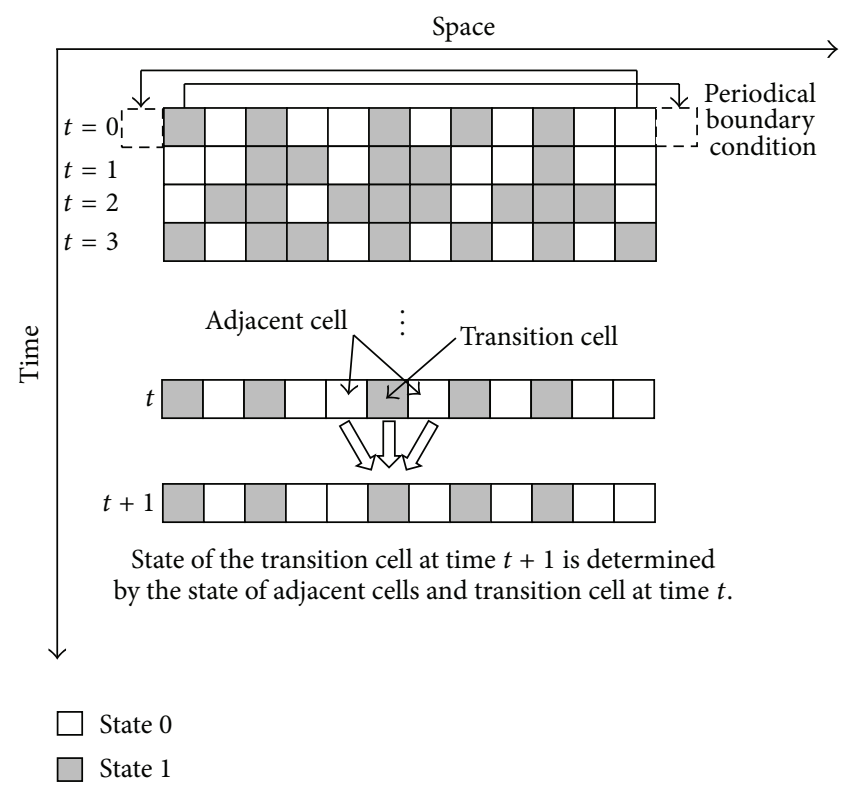

FIGURE 2: Outline of the one-dimensional cellular automata model. The horizontal direction denotes space and the vertical direction denotes time. Periodic boundary conditions are assumed and each square cell has one state, 0 or 1 , as attribute. The next cell state is determined by three cell states, that of the cell and the two adjacent cells.

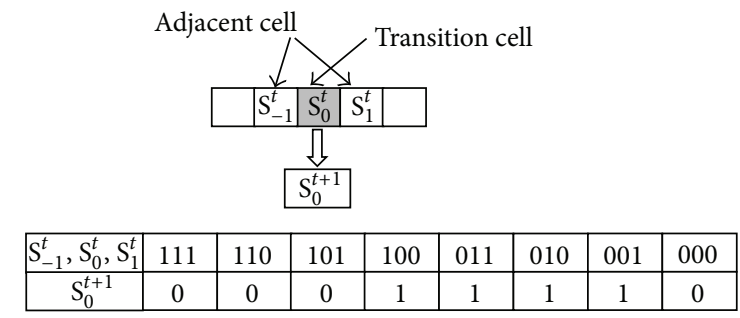

State 0

State 1

FIGURE 3: Example of the transition rule in the one-dimensional cellular automata system. The permutations of the state of three cells are $2^{3}=8$ and the state of the next step is also dyadic ( 0 or 1$)$. As a result, the transition pattern has $2^{8}=256$ arrangements.

conditions are assumed, where the right-side boundary continues to the left-side boundary and vice versa.

Each square cell has one state, 0 or 1 , as attribute. The next cell state is determined by three cell states, that of the cell and the two adjacent cells. The permutations of the state of three cells are $2^{3}=8$ and the state of the next step is also dyadic ( 0 or 1$)$. As a result, the transition pattern has $2^{8}=256$ arrangements. Figure 3 shows an example of the transition rules.

The transition rules were studied by Wolfram [5] who classified them into four groups: class 1: unchangeable pattern; class 2: periodic change; class 3: random change like chaos; and class 4: mixture of periodic or random mode. 


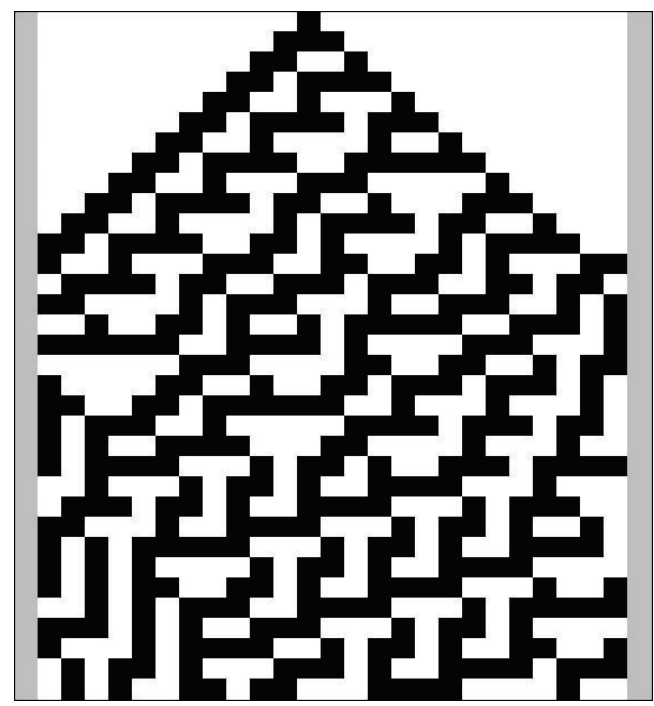

Figure 4: Example of one-dimensional cell automata. This is the result of rule 30 (00011110) that produces a shell-like pattern.

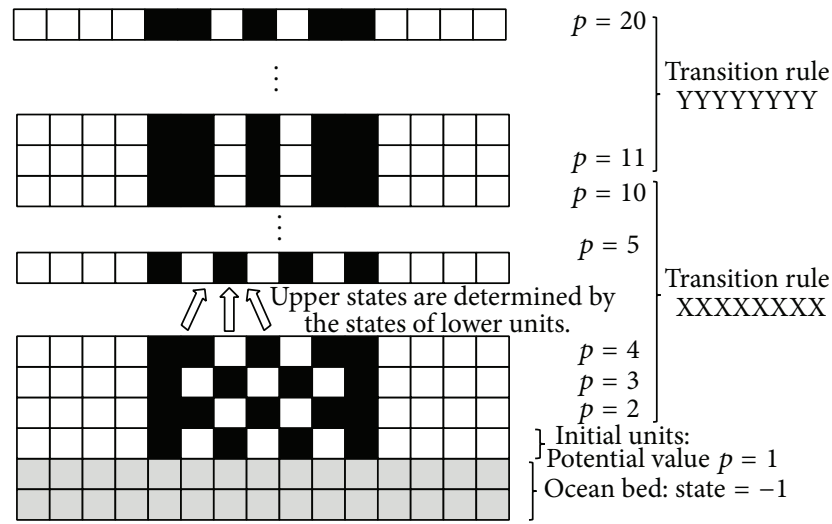

$\square$ State 0

State 1

Figure 5: Construction model of underwater space. A two-dimensional square cell grid is considered in the vertical direction. Each cell has two attributes: one is the state (ocean bed $(-1)$, marine water (0), and structural module (1)) and the other is the potential value $p$ that expresses the hierarchy from the bottom of the seabed. Under these conditions, the structural modules are piled up according to the algorithm of transition rule.

Figure 4 shows the result of rule 30 (00011110) that produces a shell-like pattern. In this study, we use the 256 arrangements to find appropriate patterns for marine structures.

2.3. Specification of Model Space. Applying the cell automata model requires modelling the underwater space. We consider a two-dimensional square cell grid in the vertical direction. Figure 5 shows the schematic overview of our model. The three-dimensional model is an extension of the two-dimensional model; however, it is not considered in this paper.

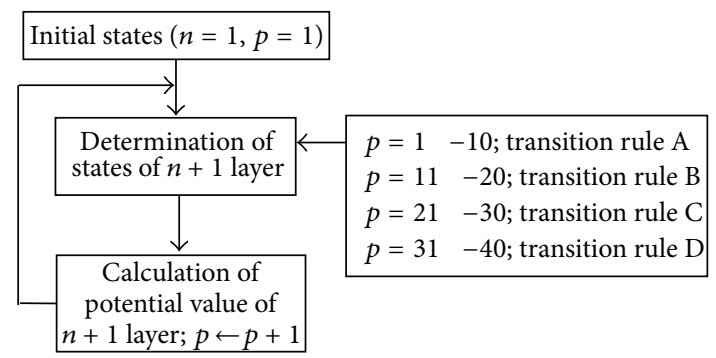

FIGURE 6: Calculation steps. The setting of each structural unit depends on the states of the three units below. Transition rules are a set of 8-bit binary numbers memorized as the design information in advance. Furthermore, the uppermost unit has the potential number of the lower units plus one. In addition, the transition rules changed every ten layers.

We assume three types of cell state: ocean bed $(-1)$, marine water (0), and structural module (1). Each cell has two attributes: one is the state $(-1,0$, or 1$)$ and the other is the potential value that expresses the hierarchy from the bottom of the seabed. In addition, each cell has some sets of 8-bit numbers as design information, which is equivalent to the cellular automata rule. As initial conditions, we consider the ocean-bed cell state and then the structural units are piled up according to the algorithm in the next section. This model replaces the time-series pattern of the one-dimensional cellular automata model with the space pattern in the sea.

2.4. Construction Algorithm. The initial conditions are as follows. The structural units (state 1) are set on the ocean bed (state -1 ) and the potential number of these units is unity. The initial structural units are the first layer of the marine structure and the second layer is constructed on the first layer. The setting of units of the uppermost layer depends on the arrangement of the lower layer. We assume the units of the next layer to sit right above and to upper right and upper left of each lower unit. The algorithm to assess the setting of each unit is based on the transition rules of the one-dimensional cellular automata model. The setting of each structural unit depends on the states of the three units below. Transition rules are a set of 8-bit binary numbers and each unit has the binary data memorized as the design information in advance.

Furthermore, the uppermost unit has the potential number of the lower units plus one. In the proposed model, the various shapes of the marine structure form by changing the transition rules every ten layers. Figure 6 shows the calculation steps.

As mentioned above, we use a simple algorithm based on the one-dimensional cellular automata model that requires a simple circuit and small memory capacity. In the proposed model, we assume four sets of 8-bit binary data, which are the blueprint data for a 40-layer structure.

2.5. Calculation Conditions. As initial conditions, the first layer of units is a ten-digit array of 0101010101. In addition, we assume two transition rules. Rule 1 is that the state of -1 (ocean bed) is constant and rule 2 is that the state (0: ocean 


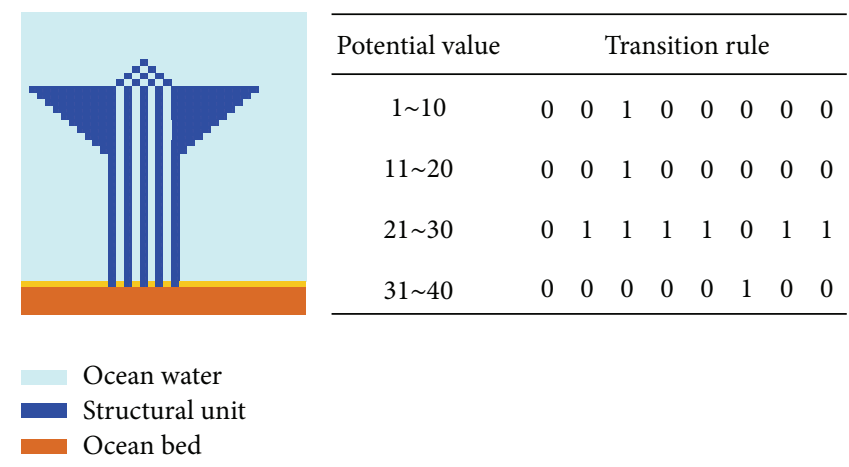

Figure 7: One calculation result. This transition rule emerged in "tower" like shape.

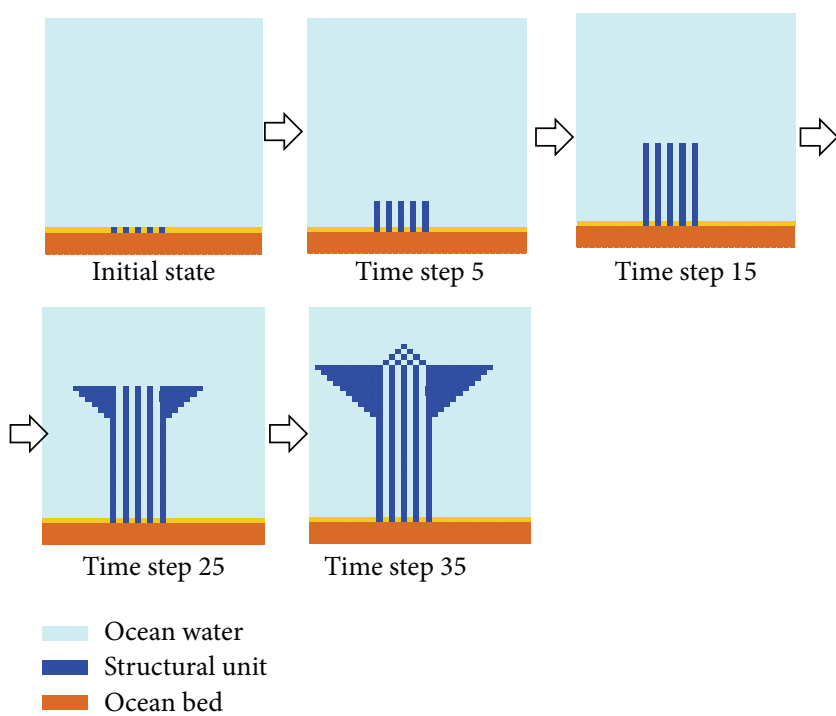

Figure 8: Calculation process of the shape in Figure 7. The structural modules are piled up according to the algorithm of transition rule.

water, or 1: structural unit) of the units of the next layer depends on the three units below (right below, lower right, and lower left unit), which combine in $2^{3}=8$ ways. The transition rules combine in $2^{8}=256$ ways and they can be expressed with 8-digit binary data.

Wolfram classified four categories of transition rules in the one-dimensional cellular automata model. Some transition rules produce a periodic pattern, whereas others produce a random or chaotic pattern. We use the transition rules that are more appropriate to design a structure.

\section{Results}

Figure 7 shows a result of the calculations. The transition rules for this result are also shown in the figure. The transition rules emerged in "tower" like shape. A variety of shapes can emerge by changing the transition rules for ten layers. This shape was determined by only 4 binary numbers described in Figure 7. Figure 8 shows the construction process of the shape shown in Figure 7.
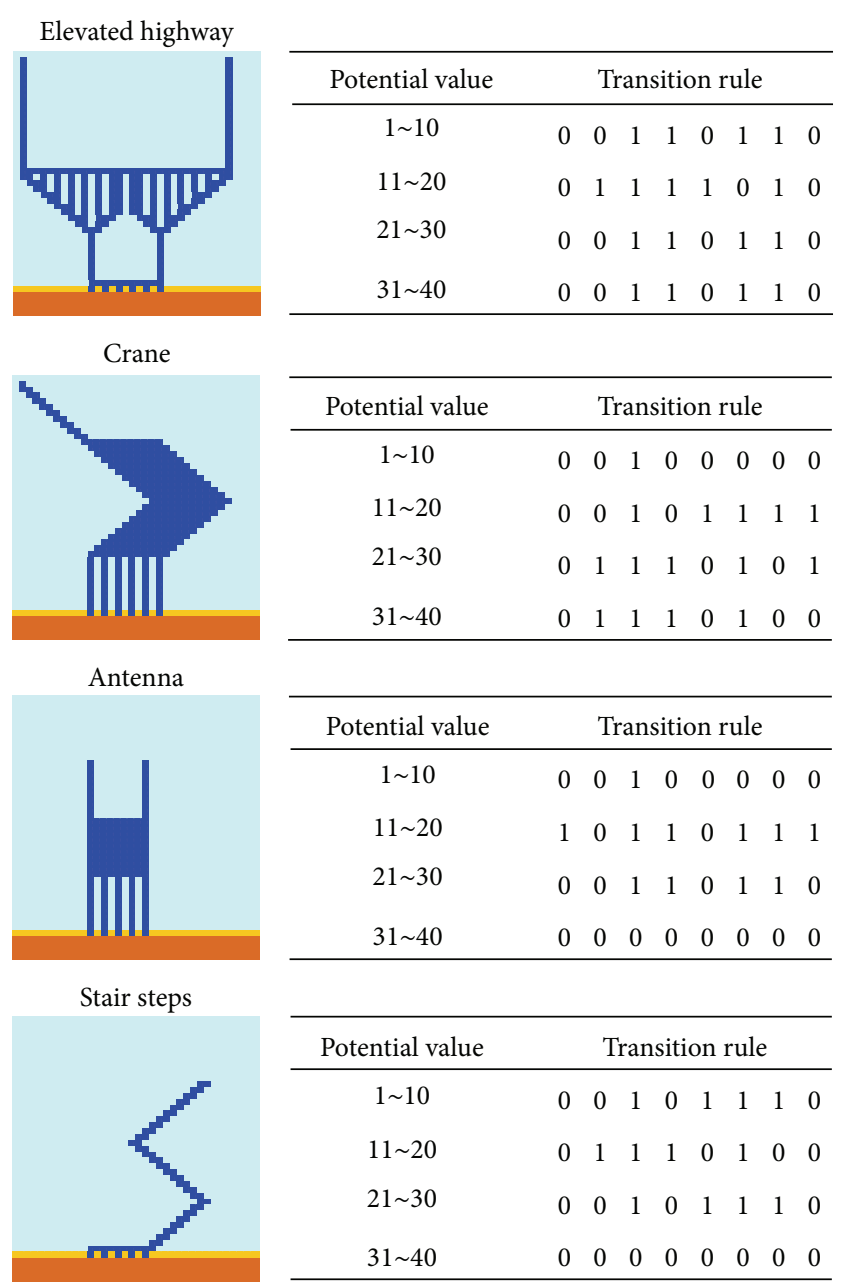

FIGURE 9: Results owing to various transition rules. It is thought that various structural shapes can be produced and they are applicable to marine structure.

The changes in the transition rules result in different structural shapes. We tried various combinations of transition rules and produced several shapes as shown in Figure 9. Names in Figure 9 are nicknames of each structure imagined from shape. From these results, it is thought that various 


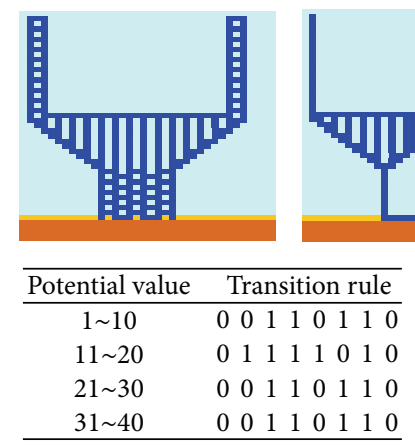

FIGURE 10: Result for variable initial conditions but the same transition rules. The initial conditions also caused slight changes in the shape of the structure under the same transition rules.

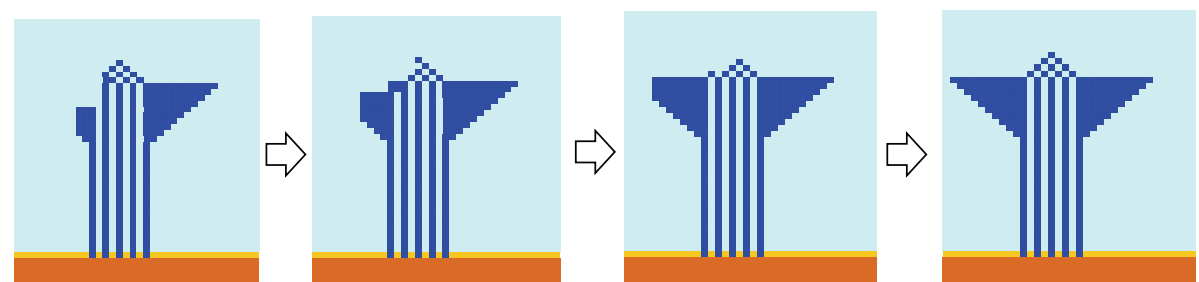

FIGURE 11: Result of the self-repairing process. The case of a damaged structure which is replaced by the grid state of the complete structure from 0 to 1 shows the capability of self-repairing a structure.

structural shapes can be produced and they are applicable to marine structure.

Figure 10 shows the results of changing the initial conditions but keeping the same transition rules. The initial conditions also caused slight changes in the shape of the structure under the same transition rules. Figure 11 shows the self-repairing process in the case of a damaged structure, which is replaced by the grid state of the complete structure from 0 to 1 . This shows that the proposed algorithm has the capability of self-repairing a structure.

\section{Discussion}

It is thought that structural units with a simple circuit, small memory capacity, and minimum communication functions can build various marine structures on the basis of onedimensional cellular automata. As a matter of course the proposed method cannot produce all shapes but only a limited variety. On the other hand, it is possible to increase the variety of shapes by taking advantage of the combinations. Furthermore, the proposed model has a self-repairing function, like a living creature, which can rebuild a damaged structure by applying the transition rules. The model also uses a smaller set of design data. For the structure in Figure 7, the difference in data volume between the proposed and a conventional model is 8 -bit binary data $\times 4$ and matrix data $29 \times 33=1190$, respectively. In addition, the amount of communication data between adjacent units also decreases with the proposed model.

\section{Conclusion}

A new self-organization algorithm is proposed to build marine structures on the ocean floor, where humans and remotely operated robots cannot operate. The algorithm is based on the one-dimensional cellular automata model that can produce various complex patterns, such as seashells, using simple transition rules. The model can also simulate the marine structure construction process using distributed cooperation control instead of central control. Furthermore, it has a self-repairing function. From a biological viewpoint, living organisms and ecosystems achieve stability and optimization because of local interactions. Using the attribute of emerging patterns in the one-dimensional cellular automata model, specific structures can be constructed on the basis of the local interaction of the transition rules without making use of complex algorithms. Apparently, in the future, the proposed model can be used to self-assemble structural units without built-in energy-consuming computer. In addition, in the future, the following need to be considered: (1) a threedimensional model of self-organizing marine structures; (2) algorithms for the carrier $\operatorname{robot}(\mathrm{s})$; and (3) prototype units with simple circuits, small memory capacity, and minimum communication functions. Such circuits may be made by IC tags or nonvolatile memory as shown in Figure 12. Furthermore, there is an approach to construct self-organizing marine structure with biochemical module called "protocell" [6]. Thus it is thought that the consideration of nanosize module is one of future approaches. 


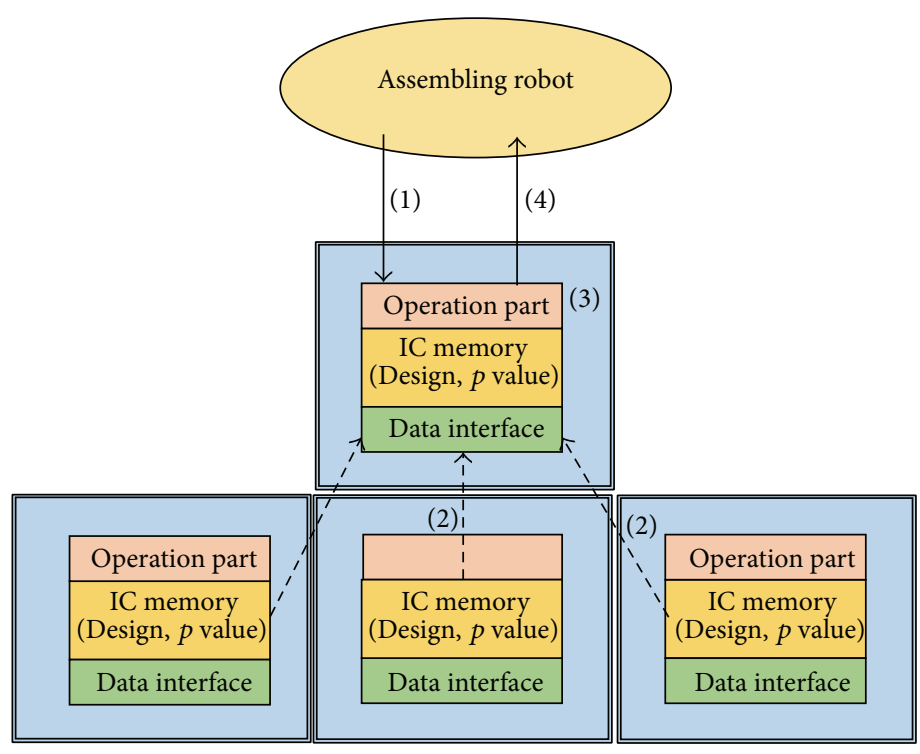

(1) Electrical power supply

(2) Transmitting potential value data and verification of units existence.
(3) Assessment of transition rule at operation part and determining the connection of unit.

(4) Feedback to assembling robot.

FIGURE 12: Example of the construction procedure of the structural units. Units need simple circuits, small memory capacity, and minimum communication functions. Such circuits may be made by IC tags or nonvolatile memory.

\section{Conflict of Interests}

The author declares that there is no conflict of interests regarding the publication of this paper.

\section{Acknowledgment}

This work was supported by a Japanese government grant (JSPS KAKENHI Grant no. 24564061).

\section{References}

[1] Y. Kato, K. Fujinaga, K. Nakamura et al., "Deep-sea mud in the Pacific Ocean as a potential resource for rare-earth elements," Nature Geoscience, vol. 4, no. 8, pp. 535-539, 2011.

[2] J. Adams, R. Bajcsy, J. Košecká et al., "Cooperative material handling by human and robotic agents: module development and system synthesis," Expert Systems with Applications, vol. 11, no. 2, pp. 89-97, 1996.

[3] E. Yoshida, S. Murata, K. Tomita, H. Kurokawa, and S. Kokaji, "Experimental study on a self-repairing modular machine," Robotics and Autonomous Systems, vol. 29, no. 1, pp. 79-89, 1999.

[4] K. Tomita, S. Murata, H. Kurokawa, E. Yoshida, and S. Kokaji, "Self-assembly and self-repair method for a distributed mechanical system," IEEE Transactions on Robotics and Automation, vol. 15, no. 6, pp. 1035-1045, 1999.

[5] S. Wolfram, "Universality and complexity in cellular automata," Physica D: Nonlinear Phenomena, vol. 10, no. 1-2, pp. 1-35, 1984.

[6] R. Armstrong, "How protocells can make 'stuff' much more interesting," Architectural Design, vol. 81, no. 2, pp. 68-77, 2011. 

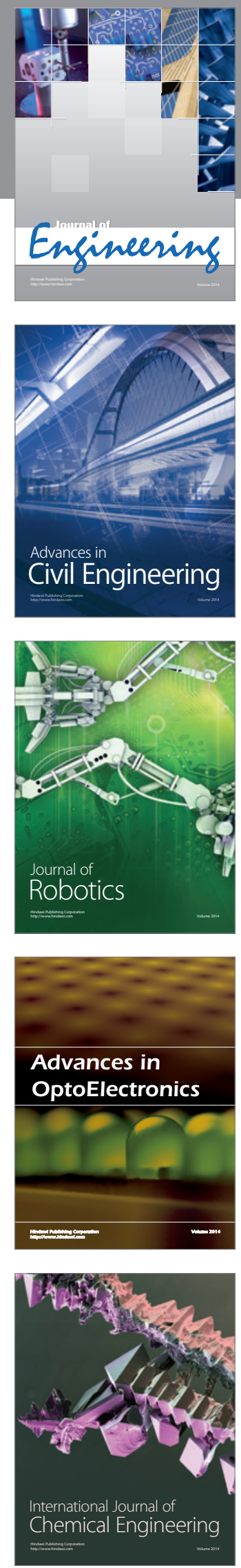

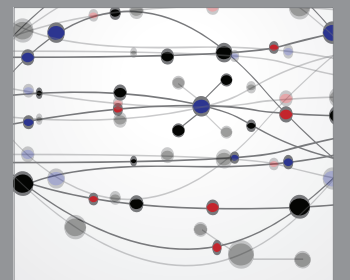

The Scientific World Journal
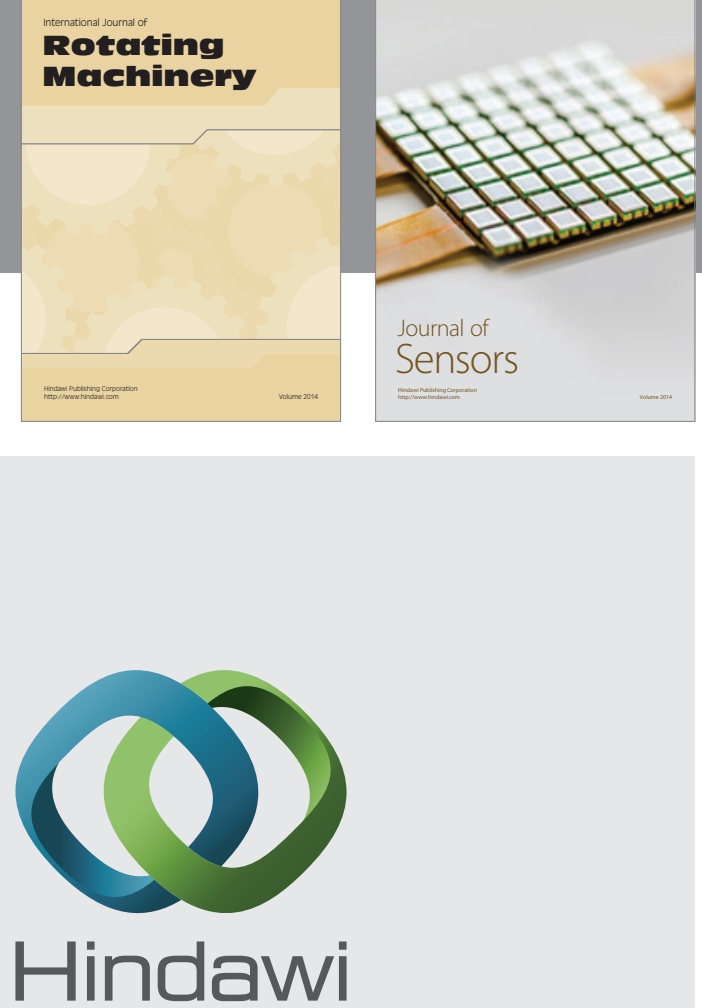

Submit your manuscripts at http://www.hindawi.com
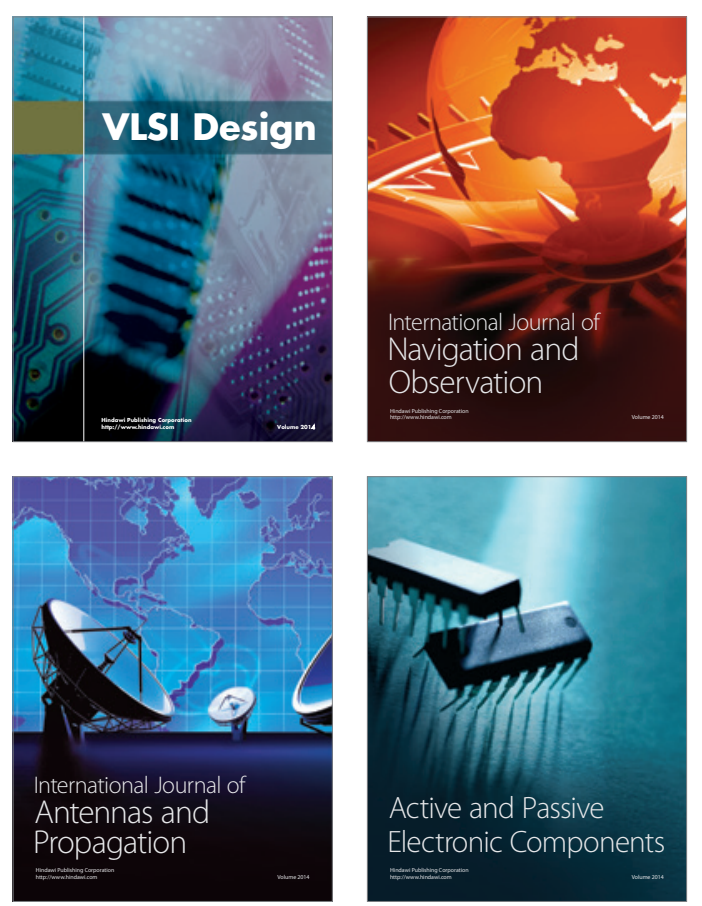
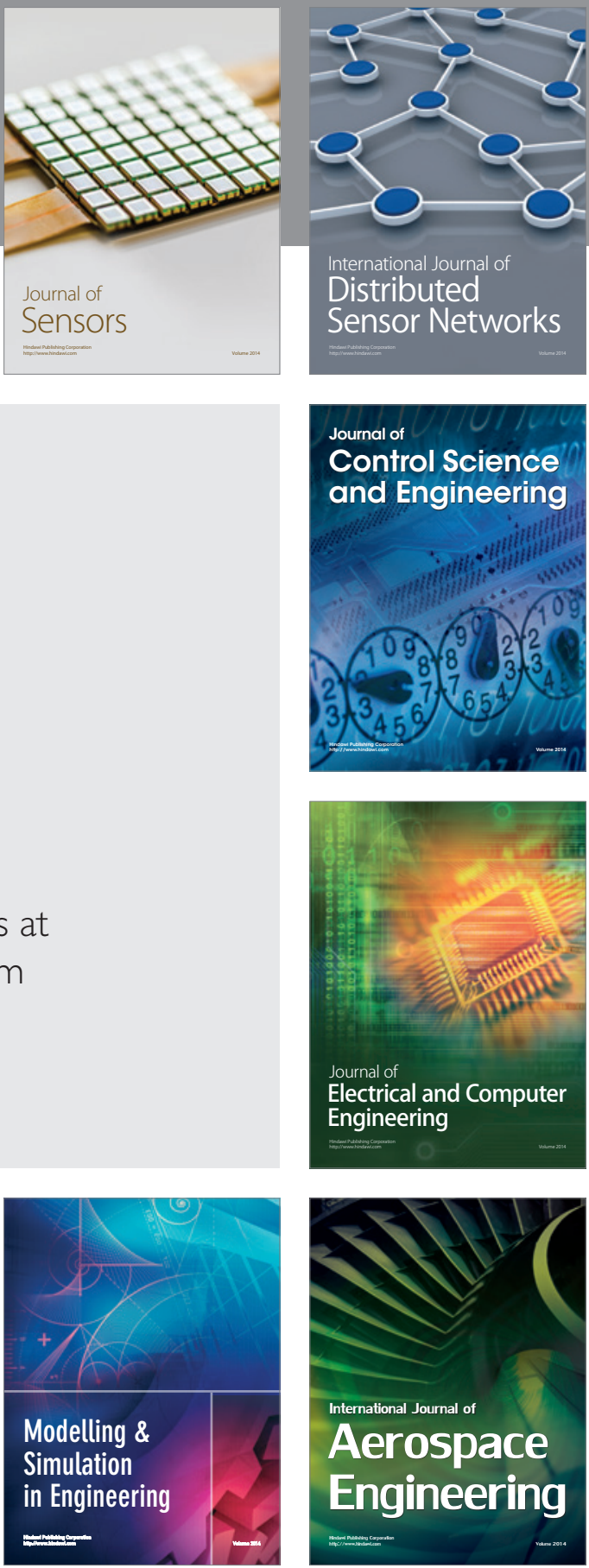

Journal of

Control Science

and Engineering
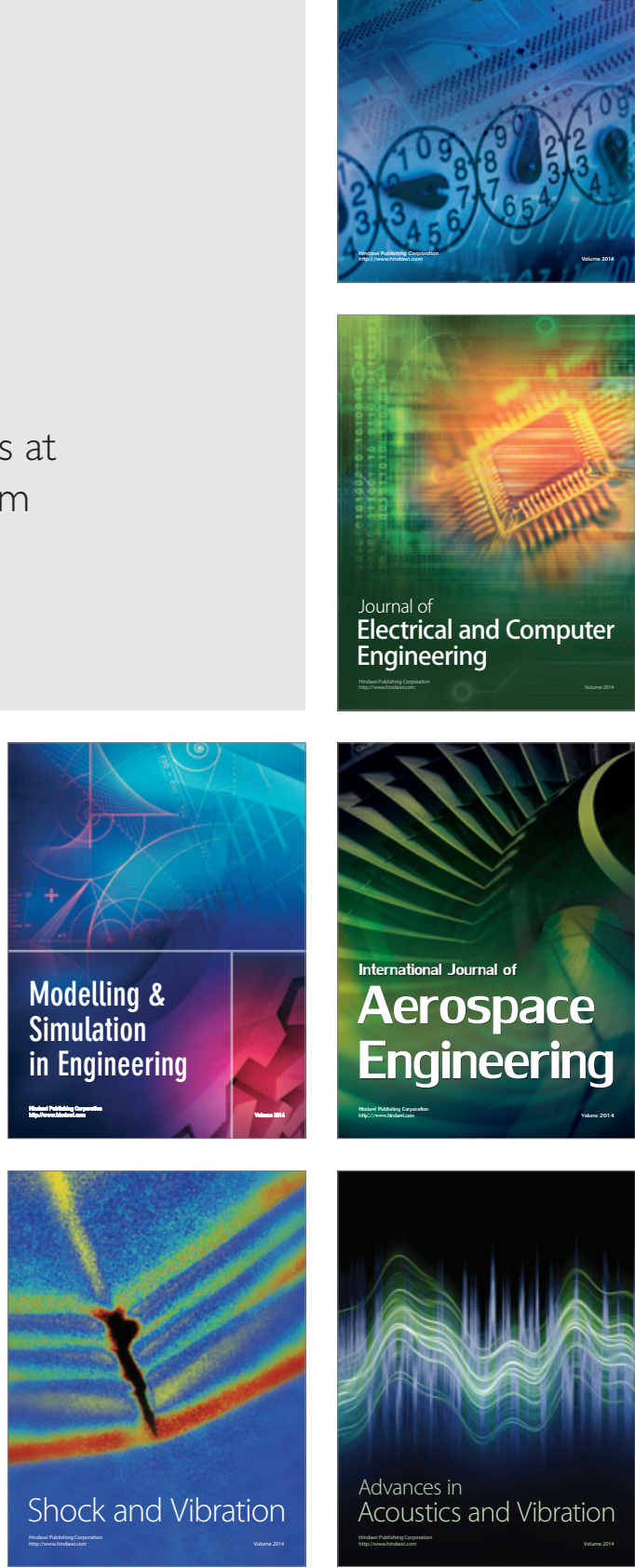